\title{
All men are created equal
}

«All men are created equal» - handschriftlich setzte der amerikanische Verfassungsvater und spätere Präsident Thomas Jefferson 1776 diesen berühmten Satz in das Gründungsdokument der ersten modernen Demokratie der Welt. Seit der «Declaration of Independence» hat dieses Wort nichts von seiner programmatischen Wirkung eingebüsst. Allerdings haben sich Verständnis und Interpretation der Begriffe «Gleichheit» und «Gleichbehandlung» mit der Zeit verändert. Das ausgehende 18. Jahrhundert assoziierte mit diesem Prinzip die Abschaffung der Sklaverei und das allgemeine Wahlrecht, also die Gleichheit vor dem Gesetz, die Chancengleichheit zur uneingeschränkten Entwicklung der unterschiedlichen Anlagen und Fähigkeiten zum «pursuit of happiness».

Im europäischen Sozialstaat des 21. Jahrhunderts haben die Begriffe eine andere Konnotation bekommen. Aus einer ursprünglich liberalen Position ist, zumindest im Umfeld der Sozialpolitik, eine egalitaristische geworden. Je unklarer die ideengeschichtlichen und ideologischen Grenzen innerhalb der heutigen Gesellschaften verlaufen, desto stärker treten hier Spannungsfelder und Widersprüche zutage. So haben wir uns mit Selbstverständlichkeit in unserem Land dem Prinzip des kompetitiven föderalistischen Wettbewerbs verschrieben, leben fern jedes gleichmacherischen Anspruchs mit den unterschiedlichsten Bildungs-, Gesundheits- und Steuersystemen, auf dass das bessere gewinne. Doch gibt es wohl andererseits wenige Bereiche unseres Lebens, in denen eine intuitive egalitaristische Haltung so ausgeprägt ist wie im Gesundheitswesen. Als Ärztinnen und Ärzte ist es zu Recht unser Anspruch, jeden Patienten gleich zu behandeln und jedem Patienten die nach unserer Kenntnis beste medizinische Betreuung zukommen zu lassen. Denselben unbedingten Anspruch auf medizinische Gleichbehandlung haben auch alle unsere Patientinnen und Patienten an uns, unabhängig davon, ob sie hohe oder tiefe Krankenkassenprämien zahlen, fiskalisch im Paradies oder einer sogenannten Steuerhölle, auf dem Land oder neben einem Universitätsspital leben. Wer in unserem Land an einem Herzinfarkt oder einem Karzinom erkrankt, wird überall die gleich optimale Behandlung erwarten, jede Ärztin und jeder Arzt wird darum bemüht sein, allen Patienten die gleiche, beste Behandlung zukommen zu lassen, und jeder Politiker wird nicht müde werden, die Qualität der medizinischen Versorgung in seinem Einflussbereich zu rühmen und die allgemeine Gleichbehandlung proklamatorisch zu garantieren.
Und doch wissen wir, dass es mit diesem Grundwert der Gleichheit im Gesundheitswesen sowohl aus einer historischen als auch aus einer geographischen Perspektive nicht weit her ist. Die westeuropäischen Sozialversicherungssysteme, in ihrem politischen Ursprung paradoxerweise eher Mittel zur Erhaltung konservativer Gesellschaftssysteme, haben ihre volle Entfaltung erst in den letzten 50 Jahren, im Lebenszeitraum der beiden letzten Generationen, erfahren. Der für uns als Selbstverständlichkeit erlebte Anspruch auf Gleichheit und medizinische Gleichbehandlung im Krankheitsfall ist also eine recht junge Errungenschaft. Leider gibt es aber keinen Grund zur Annahme, dass diese unbestritten von Dauer sein wird. In allen europäischen Staaten stecken die Sozialsysteme in einer tiefen Krise. Die Hauptgründe hierfür mögen je nach Land eine unterschiedliche Gewichtung haben, doch ein Grundkonzept ist allen gemein: Alle Sozialsysteme bauen auf der Grundstruktur des Nationalstaates auf, sind letztlich eine Staatsbürgerversicherung. Auf die abnehmende Bedeutung des Nationalstaates, auf internationale Dependenz, grenzenlosen Verkehr von Information, Geld, Waren und Menschen sind diese Systeme nicht vorbereitet. Mit der gleichen Geschwindigkeit, mit der die Globalisierung voranschreitet, stossen unsere Sozialsysteme an ihre Grenzen. Nicht nur wegen ihrer Finanzierungsstruktur, sondern auch konzeptionell. Was bedeutet heute, wenn es um Gesundheitsleistungen geht: «all men are created equal»? Wenn man genau hinhört, kommen in den politischen Diskussionen unserer Tage - nicht nur bei uns - neue Tendenzen auf. Die Egalitarismuskritik hat mittlerweile ihren Weg aus den engen Kreisen philosophischer Debattierzirkel hinaus gefunden, und in der öffentlichen Diskussion bricht zwischen den Zeilen immer deutlicher eine neue Faszination am Charme der Ungleichheit durch.

Es wird die zentrale Herausforderung der Gesundheitspolitik der kommenden Jahre sein, welches Gewicht und welche Bedeutung sie den Worten Gleichheit und Gerechtigkeit im sozialpolitischen Umfeld beimisst. Auch die Ärzteschaft wird nicht umhinkommen, in diese Diskussion einzugreifen und Stellung zu beziehen. Es mag einem gefallen oder nicht: Wirkliche Rezepte hierfür bietet die Vergangenheit keine.

PD Dr. med. Ludwig T. Heuss, Mitglied des Zentralvorstands der FMH 1994-2006 